\title{
Hybrid Systems for Robotic Navigations
}

\begin{abstract}
This paper presents a development of hardware and software of hybrid systems for autonomous robot navigation. The task for this robot is to navigate through a given field and throwing shuttlecocks through a hoop to dedicated zones. It is vital for the robot to have navigational systems for robot movement. This work develops a robot with hybrid systems using encoder, digital fiber sensor and fiber optic sensor. This work embraced mechanical parts and programming parts for robot navigations. To achieve this smooth navigation, rotary encoder was embedded on the robot tails, and left and right side of the robot. This will enable the robot to move in correct positioning. Furthermore, digital fiber sensor is used for color detection as each color gives difference response, and fiber optic sensor for detection of hindrance or fence. The hybrid approach is observed to be competent for the robot to navigate autonomously and effectively in unknown environments. It has been shown that the motion of the robot can be operated as target conditions and shows that color and hindrance or fence plays an important role in navigation system.
\end{abstract}

